\title{
Enhancing QoS Through Dynamic and Fare AP Selection in a Wireless LAN
}

\author{
Fakhar Uddin Ahmed \\ Department of Computer Science, LCB College, Guwahati, Assam, India \\ Email: fakharuddin4@rediffmail.com \\ Shikhar Kumar Sarma \\ Department of Information Technology, Gauhati University, Guwahati, Assam, India \\ Email: sks001@gmail.com
}

\begin{abstract}
The IEEE 802.11 WLAN is primarily used for web browsing which belongs to the category of nonreal time application. But the demand of real time applications like VOIP and video conferencing has become very much common to such WLAN. With IEEE 802.11e Mac protocol it is possible to improve the QoS for both real and non-real time traffic by service differentiation. To ensure efficient utilization of the radio resources and enhanced QoS the load imbalance should be resolved among APs from different BSSs. In large scale WLAN inter AP communication mechanism can be employed along side the current admission controller under EDCA. Beside service differentiation inter AP differentiation based QoS management can lead to efficient utilization of radio resources by moving STAs from heavily laded to a less loaded AP and ensure better QoS for all types of traffics. In this paper we propose a dynamic and fair AP selection mechanism to improve the QoS in a WLAN. The simulations have been carried out with NSv2.34.
\end{abstract}

Index Terms - EDCA, QoS, Contention Window, VoIP, AC.

\section{INTRODUCTION}

In the last few years the IEEE 802.11 [1] technology has become very interesting. The significant performance benefits of wireless LANs have made them an ideal networking platform for offices, homes, and public places like airports, malls, hotels, etc. The key challenges of such WLAN [2] are optimal selection of APs (Access Point) and efficient utilization of available radio resources [3]. Moreover real-time applications, such as VoIP (Voice over IP) [2] and video conferencing are considered to be next killer applications and therefore the QoS for real time traffic should be maintained at the same time. In order to guarantee the QoS for IEEE 802.11e, the IEEE 802.11 Task Group has proposed a new protocol, IEEE 802.11e. In IEEE 802.11e, HCF [2] (Hybrid Coordination Function) is defined which corresponds DCF (Distributed Coordination Function) and PCF [2] (Point Coordination Function) in 802.11. HCF is composed of EDCA
(Enhanced Distributed Channel Access) which controls the channel access in contention period and HCCA (HCF Controlled Channel Access) which controls the channel access in non-contention period. HCF is backward compatible with the legacy MAC. Even if IEEE 802.11e has standardized QoS enabled MAC service, but still we need mechanisms to admit and reject traffics for various TCs (Traffic category) to maintain QoS. Admission control plays an important role in supporting QoS for IEEE 802.11 [1] based WLANs. The admission controller function accepts or rejects resource reservation request, based on utilization. The Admission controller's job is to limit the amount of traffic can be admitted into a particular access category ensuring that the QoS [3] of the existing flows is not affected. With EDCA MAC parameters and admission controller we can achieve improved QoS for real and non-real time application but does not guarantee efficient utilization of radio resources in certain situations. In particular there might be no competing real time applications engaging in an AP but less chances for non-real time traffic to access the wireless medium. There might be situations also where more real time applications exist under a particular AP which may lead to lower throughput and low QoS performance in that AP where upon other APs in the ESS (Extended Service Set) are sitting idle. In this paper we present an inter AP differentiation based QoS management for efficient utilization of radio resources balancing AP loads in a such WLANs and ensure better QoS for all types of traffics. The rest of the paper is organized as follows: in section 2, we outline the IEEE 802.11e and Admission Controller, section 3 describes our proposed mechanism, section 4 includes Simulation and result discussion and section 5 includes conclusion and future work.

\section{IEEE 802.11E AND ADMISSION CONTROLLER}

In this section we describe the IEEE 802.11e channel access method and admission controller under EDCA [3].

\section{A. $E D C A$}

IEEE 802.11e [3] has recently proposed a new 
contention-based channel access method called enhanced distributed channel access (EDCA). The

EDCA [3] introduces the concept of access categories. Each station has four kinds of access categories, or priority levels to differentiate the channel access on the wireless medium. With EDCF, high priority traffic has a higher chance of being sent than low priority traffic: a station with high priority traffic waits a little less before it sends its packet, on average, than a station with low priority traffic. This is accomplished by using a shorter contention window $(\mathrm{CW})$ and shorter Arbitration Inter frame Space AIFS) [4]. Each access categories (AC) contends for the medium with the same rules as the standard DCF (i.e. wait until the channel is idle for a given amount of inter frame space IFS, and then access/retry following exponential backoff rules). The access probability differentiation is provided by using different AIFSs instead of the constant distributed IFS (DIFS) used in DCF, and ii) different values for the minimum/maximum.

CWs to be used for the backoff time extraction. Then, each $\mathrm{AC}$ is specified by the values $A I F S[A C], \operatorname{Wmin}[A C]$, and $C W \max [A C]$. The AIFS[AC] [3,4] values each differ for an integer number of backoff slots. In particular,

\section{AIFS $[$ AC $]=$ AIFSN $[$ AC $] \cdot$ aSlotTime + aSIFSTime}

Where $A I F S N[A C]$ is an integer greater than 1 for normal stations and greater than 0 for APs. Table 1 shows the default values of the channel access parameters defined in

EDCA for the four ACs (BK = background, $\mathrm{BE}=$ best effort, $\mathrm{VI}=$ video, $\mathrm{VO}=$ voice $)$. Note that these parameters are not fixed: in each beacon frame, the access point (AP) broadcasts the values chosen for each AC. Indeed, these values may also be dynamically adapted according to network conditions. Obviously, the smaller the AIFSN[AC] and $C W \min [A C]$, the higher the probability of winning the contention with the other ACs. the smaller the AIFSN[AC] and CWmin[AC], the higher the probability of winning the contention with the other ACs. When more than one AC of the same station expire its backoff counter, a virtual collision occurs, and the highestpriority packet among the colliding ones is selected for actual transmission on the radio channel. Fig. 1 shows the IEEE 802.11e channel access method.

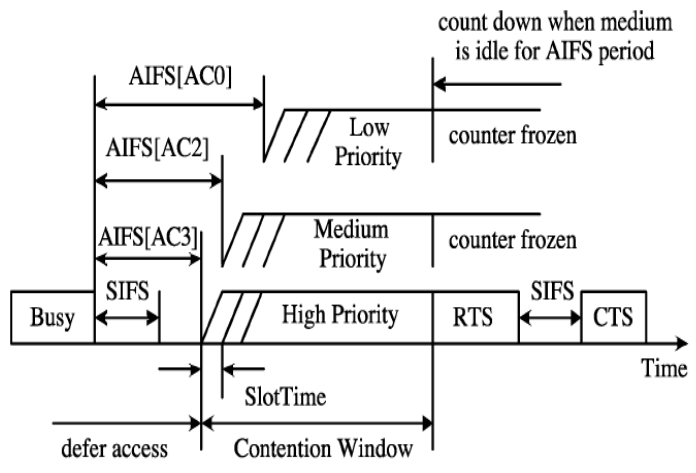

Figure 1. IEEE 802.11e Channel Access

\section{B. Admission Controller under EDCA}

Admission controller is needed when a QSTA requests for an adequate amount of time required to access the wireless medium. In 802.11 EDCA a STA is assumed to send an ADDTS (Add traffic specification) frame to the AP. The ADDTS frame contains traffic category and traffic specification (TSPEC) corresponding to the specific application. The TSPEC contains the following information.

- Mean Data Rate: the average bit rate for packet transmission, in bits per second (bps)

- Delay Bound: the maximum delay allowed to transport a packet across the wireless interface (including queuing delay), in milliseconds;

- Maximum Service Interval: the maximum time allowed between neighbor TXOPs allocated to the same station, in microseconds;

- Nominal MSDU Size: the nominal size of a packet, in octets;

- $\quad$ Minimum PHY Rate: the minimum physical bit rate assumed by the scheduler for calculating transmission time, in bps.

In [4] Chen et al. implemented CAC algorithm taking channel utilization and delay as a performance metrics for QoS required by real time traffic. The algorithm works by monitoring channel busyness ratio of the real time applications. Before initiating a real-time flow of priority $i(i=2$ or 3$)$, a node must send an ADDTS [4] (add traffic stream) request to the coordinator. Upon receiving the ADDTS, the controller associates the flow with the appropriate $\mathrm{AC} i$ and mean channel utilization $\left(\mathrm{Cu}_{i \text { mean }}\right)$ is evaluated as

$\mathrm{c} u_{i, \text { mean }}=\mathrm{R}_{\text {mean }} / \mathrm{L}\left(\mathrm{T}_{\text {suc }}\right)$

Where $\mathrm{T}_{s u c}=R T S+C T S+D A T A+A C K+3 S I F S+A I F S$ is the successful transmission time in RTS/CTS mode, $\mathrm{R}_{\text {mean }}$ is mean data rate and $\mathrm{L}$ is the nominal MSDU size belonging to a particular access category. In [4] they set a quota on channel utilization by real time traffic denoted by $U_{r t}$, to $80 \%$ of the maximum channel utilization, denoted by $U_{\max }$ and the best effort traffic is entitled to $20 \%$ of the channel utilization so it is operational at all time. When the QAP receives an ADDTS frame, the controller associates the flow with the appropriate AC $i$ and obtains $\mathrm{u}_{i \text {,mean }}$ using (1). The flow can be admitted if the following conditions holds true.

$\left.\begin{array}{l}u_{A, \text { mean }}+u_{i, \text { mean }}<U_{r t} \\ u_{A, \text { peak }}+u_{i, \text { peak }}<U_{\max }\end{array}\right\}$

\section{PROPOSED MECHANISM}

The admission controller and channel access method described in section 2 improves QoS for real time 
application in an AP due to reservation in the channel access transmission time by setting large contention window and shorter AIFS values. But reservation in channel access does not ensures efficient channel utilization because traffics of higher category may not exist in particular situations and also by accommodating more traffics of higher category can affect other traffics QoS. This paper proposes a new mechanism for associating traffics to an AP in a way to improve the QoS $[5,6]$ in a WLAN. This mechanism is an enhancement of the admission controller in the sense that admission controller will have to monitor QoS performance in the AP due to all accommodated association and also traffic load conditions in other APs within the ESS for resolving the load anomaly [7]. This can be possible by making inter AP communication. Primarily our proposed mechanism (EAC) Extended Admission Controller consists of two modules the QoS Monitoring Program (QMP) and IAPC (Inter AP Communication). The QMP module monitors the traffic and QoS level in a QAP in a particular point of time by analyzing the various parameters like Number of STAs associated, Channel utilization etc... The QMP module is a mediator between the Admission Controller and IAPC. The IAPC module is triggered by the QMP module when the QoS performance or maximum channel allocation occurs in a QAP. When indication from the Admission Controller is given to the QMP for such crisis of QoS performance it may call IAPC to resolve the same by negotiating with other QAPs. If accommodation can be given in some other QAP within the ESS QMP reports it to the Admission Controller and consequently the Admission Controller can send some suggestions to the QSTAs about the learned situation of the traffic and QoS level and thus QSTAs may take appropriate and optimal decision in the selection or reassociation of Access Points. We describe both the modules in the following subsections. For EAC we consider the Metrics Channel Utilization, Channel busyness ratio, number of STAs associated and available admission capacity in an AP. The definitions of each term is explained below.

- Channel Utilization ( $\left.\mathbf{R}_{s}\right)$ : It is the ratio of successful transmission periods to the total time.

- Channel Busy Ratio ( $\left.\mathbf{R}_{\mathbf{b}}\right)$ : It is the amount of time the channel is determined to be busy to the total time. The channel busyness time consists of periods of both successful transmissions and collisions.

- Channel Utilization ( $\mathbf{R}_{\mathbf{s}}$ ): It is the ratio of successful transmission periods to the total time.

- The Available Admission Capacity: AAC contains an unsigned integer that specifies the remaining amount of medium time available in units of 32 microseconds per 1 second.

\section{A. $Q M P$}

In view of the metrics mentioned in section 2.2, the QMP module will monitor the values of all metrics $R_{b}$, $\mathrm{R}_{\mathrm{s}}$, AAC, NoSTA etc. for the efficient utilization of radio resources. The QMP module will not only monitor the traffic load level of a QBSS but the other QBSS as well within the ESS with the help of IAPC module. The IAPC module is triggered in two particular cases by the QMP module.

Case 1: QMP periodically monitors the Channel Busy Ratio, $\mathrm{R}_{\mathrm{b}}$ in the QBSS. And if $\mathrm{R}_{b} \geq \mathrm{U}_{\max }$ i.e. QoS level is below some certain threshold value it invokes IAPC for negotiating other QAPS and relegating some traffic having lowest level of QoS to some other QAP relatively less loaded.

Case 2: If certain flow cannot be accommodate due to maximum association in that QAP (using (2)) then QAP may call QMP for getting some suggestion from the IAPC so that an optimal response can be given to the requesting QSTA. The pseudo code of the QMP module to measure the traffic load level is presented as follows.

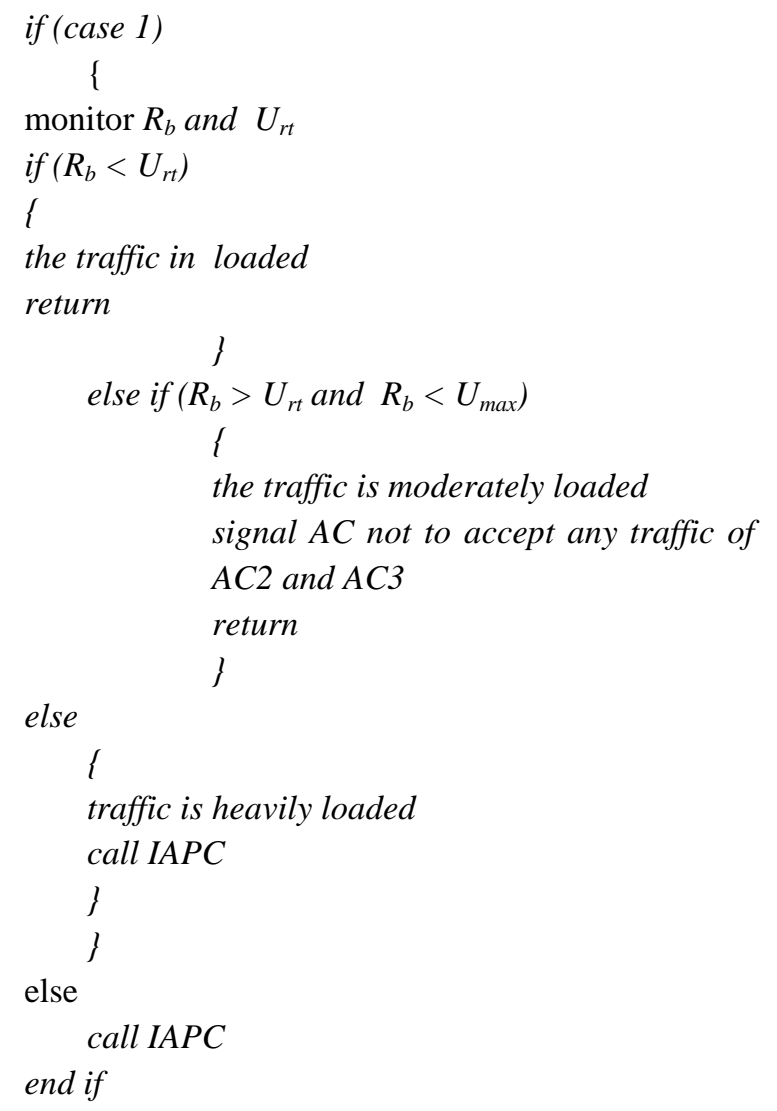

\section{B. IAPC}

In the design approach of IAPC, each AP exchanges information's with other APs. Each AP maintains an AP table info that contains AP's SSID (Service Set identifier), AP's IP Address, AP's MAC Address, Number of associated Stations, Channel busy ratio, Available admission capacity. The algorithm for IAPC is as given below here. 


\section{AP gathers information of other APs around it}

2. AP logs the informations of other AP which have minimum number of STA and narrows down candidate APs

if (Number of candidate AP is less than 1)

return from IAPC without any selection

else if (Number of candidate AP is greater than 1)

goto 3

else if (Number of candidate AP is equal to 1 )

goto 5

end if

3. AP logs the available admission capacity in other $A P$ and narrows down candidate APs which have maximum ATC If (Number of candidate AP is greater than 1)

goto 4

else if (Number of candidate AP is equal to 1)

goto 5

end if

4. select one AP form the candidate AP set which have lowest $R b$ and goto 6

5. end selection and return from IAPC with selected APs AP_ID

\section{EAC Algorithm}

For efficient utilization of radio resources EAC periodically monitors the Channel busy ratio $\mathrm{R}_{b}$. If $\mathrm{R}_{b}<$ $\mathrm{U}_{r t}$ the channel is not regarded to be loaded. When $\mathrm{U}_{r t}<$ $\mathrm{R}_{b}<\mathrm{U}_{\max }$ the channel is regarded to be moderately loaded and if $\mathrm{R}_{b}>=\mathrm{U}_{\max }$ the channel is regarded to be heavily loaded and Inter-process AP communication (IAPC) is triggered. At an optimal point $\mathrm{R}_{b}$ is almost equal to $\mathrm{R}_{s}$. The principle aspect of EAC is to keep $\mathrm{R}_{b}$ close to $\mathrm{R} s$. This proposed algorithm waits for ADDTS request from STAs with certain bandwidth requirement of AC $i$ and perform the following steps.

1. for each flow of AC [i] from STAs do

2. calculate Cost of Association using eq.(1)

3. if (QoS is ok due to new flow) using eq.(2) accept the new flow

send successful ADDTS response to STA update the new TSPEC in the database else

call IAPC

if (There is a solution) send ADDTS suggestion to STA

else

send ADDTS response to STA

with retry status

end if

end if

end for

Copyright @ 2014 MECS

\section{SIMULATION AND RESULT DISCUSSION}

We conducted our simulation under GNU/Linux Operating system (OpenSuse11) using Network simulator 2.34[8].This simulation package includes IEEE802.11e patch [9]. The simulation area is set to area $200 m \times 200 \mathrm{~m}$. In the simulation we place two APs, AP1 is placed in $100 m \times 90 m$ and AP2 is placed in $140 m$ $\times 50 \mathrm{~m}$. We choose IEEE $802.11 \mathrm{~b}$ PHY layer, and the PHY data rate is set 11 Mbps. EDCA parameters [3] used in the simulation are shown in the table 1 . In this simulation we consider two cases. In case 1 we add 3 STAs first and eventually increase the STAs by 3 up to a maximum of 24 STAs in different traffic categories (Voice, Video and Best effort). In case 2 simulations we move some real traffic from AP1 to AP2 based on our proposed algorithm. Then sensors with packets of the next priority level will join in transmission when last transmission time is over.

TABLE 1. EDCA PARAMETERS

\begin{tabular}{|c|c|c|c|c|}
\hline Traffic & $\begin{array}{c}\text { Transport } \\
\text { Protocol }\end{array}$ & CWmin & CWmax & AIFSN \\
\hline Voice & UDP & 3 & 7 & $30 \mu$ \\
\hline Video & UDP & 7 & 15 & $30 \mu$ \\
\hline $\begin{array}{c}\text { Best } \\
\text { Effort }\end{array}$ & UDP & 15 & 1023 & $30 \mu$ \\
\hline
\end{tabular}

For evaluating the performance we consider the following metrics.

- Throughput: Throughout can be defined as the number of bits successfully received by the receiver divided by the total transmission period of time in seconds.

- Packet Loss: Packet loss is defined for a receiving mobile station as the number of packets lost or dropped during transmission

\section{A. Simulation Results in Case 1}

Fig. 2 and Fig. 3 show throughput and Packet Loss experienced by each access category of AP1 in case 1 simulation. From simulation result it is clearly understood that EDCA provides service differentiation for traffic of different categories. When the number of STA is kept under 5 all the data flows have equal channel access. But in case of increased number of STA the channel is reserved for high traffic categories and QoS is ensured for real time traffic, but best effort traffic suffers from performance degradation. As shown in Fig. 2 the packet loss for best effort traffic starts when number of STA is 5 and goes high gradually. This is due to the fact that the best effort flow has lowest priority. On the other hand real time traffics have almost consistent performance when the number of STA is kept under 12. But there has been a significant drop in throughput and packet loss as number of traffic increases Fig. 3 shows the packet loss experienced by each traffic category. For voice traffic upto 13 STAs there is no 
packet loss due to smallest contention window. But with increasing traffics the packet loss increases and we have found $34 \%, 47 \%$ and $71 \%$ of packet loss for voice, video and best effort traffic respectively.

\section{B. Simulation Results in Case 2}

In case 2 simulations we move some of the real time traffic experiencing low throughput and high packet loss from AP1 to AP2 using the proposed mechanism of AP selection. The simulation results show improved performance of the traffics moved from AP1. The results are shown in Fig. 5 and Fig. 6. Consequently we again record the throughput and packet loss of the remaining STAs of AP1 and result graphs with improved performance is shown in Fig. 7 and Fig. 8.

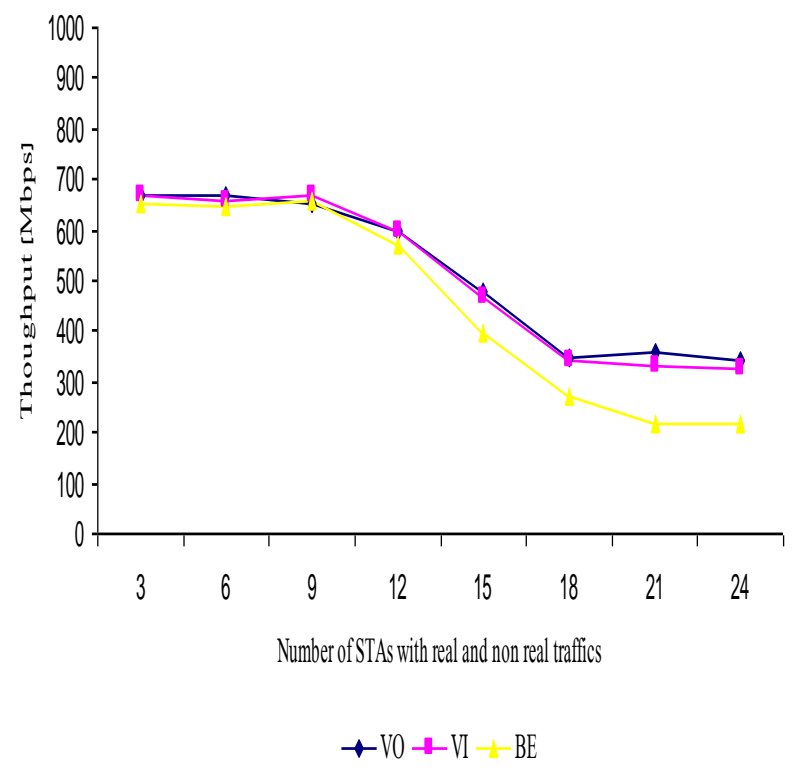

Figure 2. Throughput for case 2 simulation in AP1

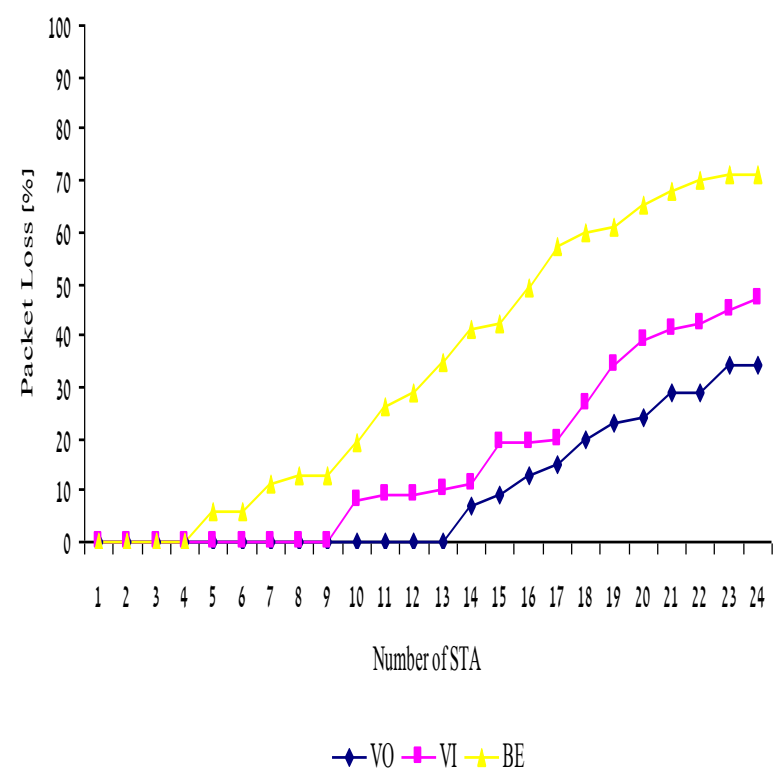

Figure 3. Packet loss for case 1 simulation in AP11

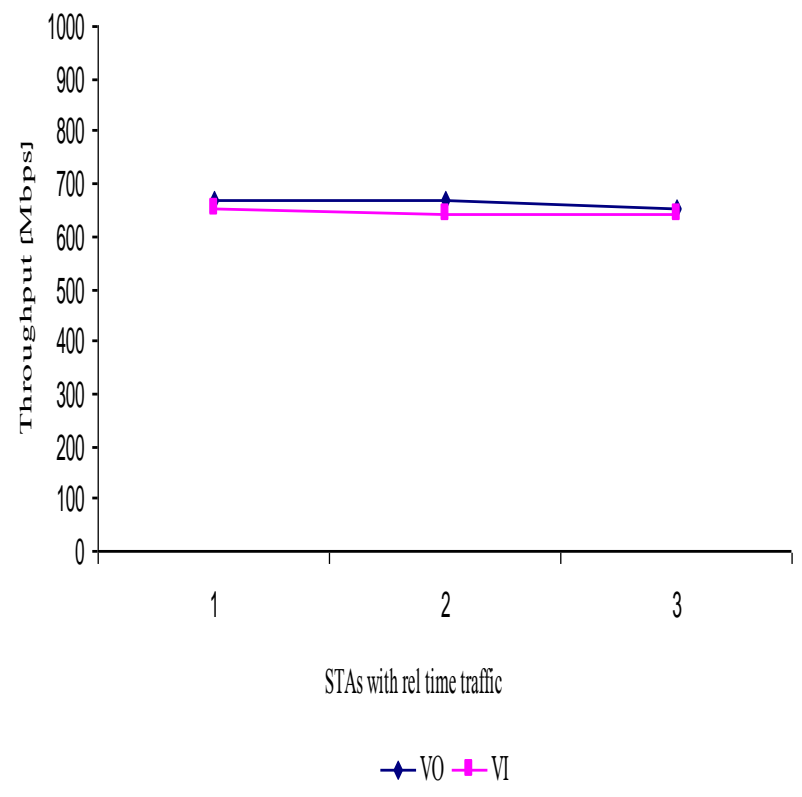

Figure 4. Throughput for case 2 simulation in AP2

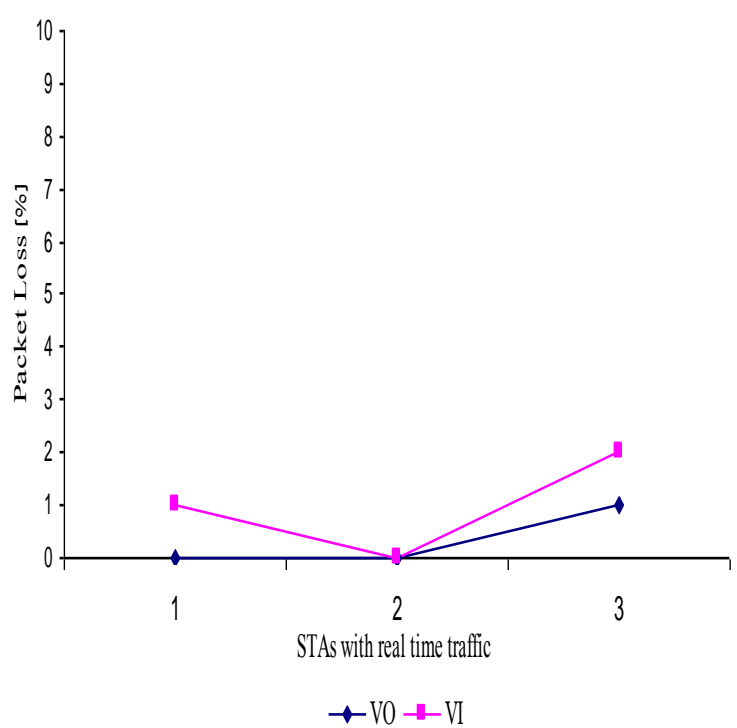

Figure 5. Packet loss for case 2 simulation in AP2 


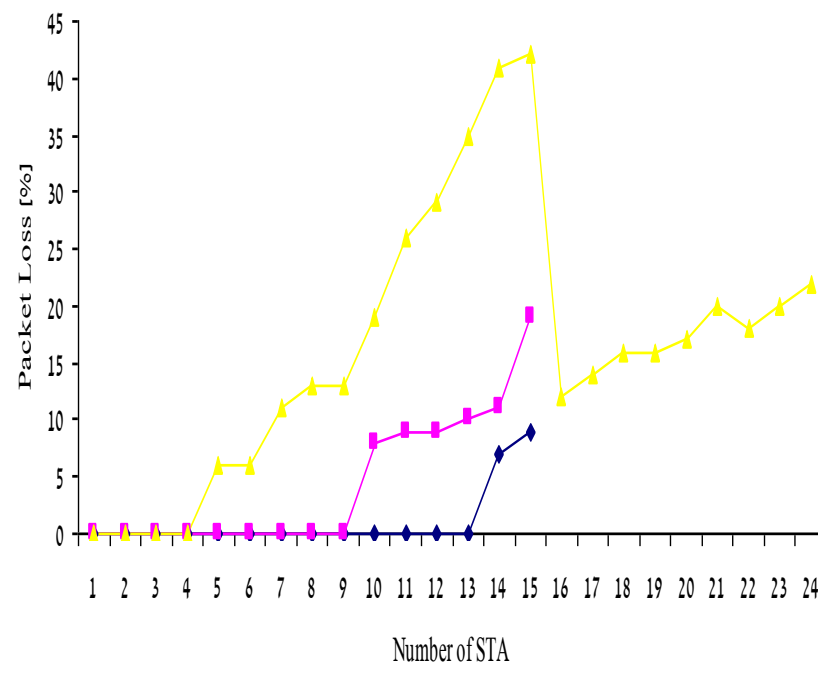

$\rightarrow-\mathrm{VO}-\leftarrow \mathrm{VI} \rightarrow \mathrm{BE}$

Figure 6. Throughput for case 2 simulation in AP1

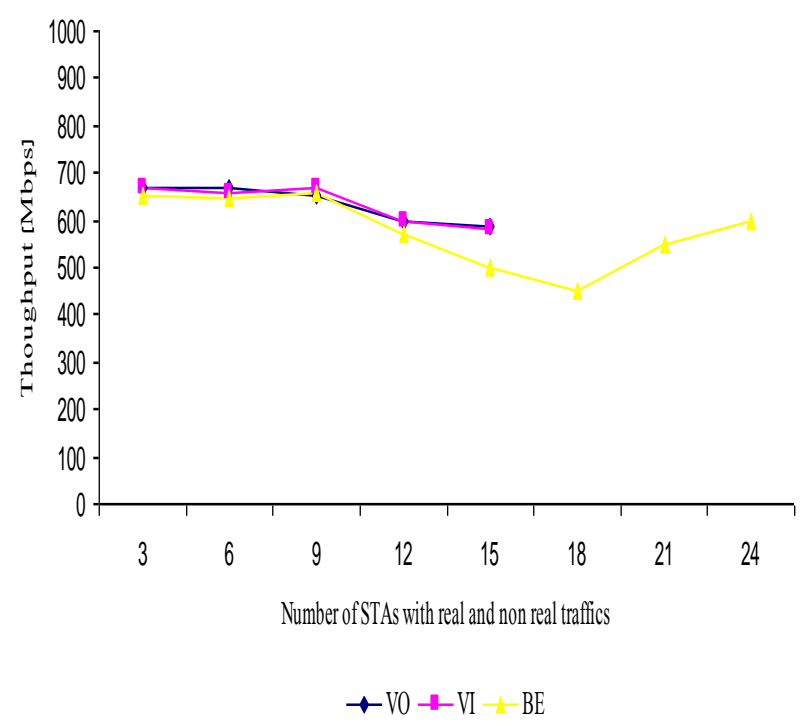

Figure 7. Packet loss for case 2 simulation in AP1

\section{CONCLUSION AND FUTURE WORK}

The main objective of this paper was to design a dynamic and fair AP selection mechanism in an IEEE 802 .11 WLAN. The major contribution of this paper is the extension of existing Admission Controller mechanism. A totally new software mechanism has been proposed to select appropriate APs for the mobile stations in a way to improve QoS performances. In the proposed software architecture we have taken the admission controller and two of the newly modules are added namely the IAPC and QMP. Alongside the EDCA parameters and admission controller, this two module will act to balance the loads within the ESS by need based association between Mobile Stations and Access Points.
The new EAC mechanism clearly improves the performance of a BSS and it can even improve the overall performance of the wireless LAN because the Inter AP negotiation helps one particular AP to know the load situations of other APs and in the crisis situations it can easily transfer and accommodate certain traffics to utilize the radio resources.

The proposed mechanisms do have some room for further development. The QMP and IAPC modules are the major contributions of this paper. The QMP module further can be worked upon to make it a robust one for monitoring QoS level in the Basis Service Sets. Further, user level priorities may configured with this mechanism whereby a portion of the channel bandwidth can be reserved to some specialized persons no matter what kind of traffic category it is possessing.

\section{REFERENCES}

[1] S. Choi, J. Prado, S. Mangold, and S. Shankar, "IEEE 802.11e contention-based Channel Access (EDCA) performance evaluation," in Proc. IEEE ICC, May 2003.

[2] "Wireless LAN Medium Access Control (MAC) and Physical Layer (PHY) Specifications: Medium Access Control (MAC) Quality of Service (QoS) Enhancements", IEEE Std. 802.11e/D8.0, Feb. 2004.

[3] X Shojiro Takeuchi, Kaoru Sezaki and Yasuhiko Yasuda "Access Point Selection Strategy in IEEE802.11e WLAN Networks".

[4] X. Chen, H. Zhai, X Tian, and Y. Fang, "Supporting QoS in IEEE 802.11e Wireless LANs", IEEE Transactions onWireless Communications, Vol. 5, Issue 8, Page(s): 2217 -2227, August 2006.

[5] Mobility and QoS of 802.11 and 802.11e Wireless LAN Standards By Fedoua Didi1, Houda Labiod2, Guy Pujolle3, and Mohamed Feham1.

[6] Mobility and QoS of 802.11 and 802.11e Wireless LAN Standards By Fedoua Didi1, Houda Labiod2, Guy Pujolle3, and Mohamed Feham1.

[7] O. Brickley, S. Rea, and D. Pesch, Load balancing for QoS enhancement in IEEE802.11e WLANs using cell breathing techniques," in IFIP Mobile and Wireless Communication Net- works Conf., Marrakech, Morocco, Sept. 2005.

[8] The Network Simulator ns http://www.isi.edu/nsnam/ns/

[9] IEEE802.11e patch http:// www.tkn.tuberlin.de/research/802.11e_ns2/

Fakhar Uddin Ahmed M.Sc PhD received his master in Computer Science in 2008 and $\mathrm{PhD}$ in 2013 from Gauhati Univesity, Assam, India. He is an Assistant Professor of Computer Science at LCB College, Guwahti Assam, India since 2008. His area of research 
is Computer Networks, particularly Wireless Networks (802.11).

Shikhar Kumar Sarma, M.Sc. PhD is a Professor and Head of the Department of Information Technology at Gauhati University, Assam, India, and Chief
Investigator, Language Technology Development Projects (NE Wordnet, CLIA); sponsored by ministry of Communication and IT, Govt. of India. He was also associated with IIT-Guwahati and Debub University, Ethiopia. His research area primarily includes Computer Networks, NLP, and Expert Systems.

How to cite this paper: Fakhar Uddin Ahmed,Shikhar Kumar Sarma,"Enhancing QoS Through Dynamic and Fare AP Selection in a Wireless LAN", IJCNIS, vol.6, no.2, pp.23-29, 2014. DOI: 10.5815/ijcnis.2014.02.04 